\title{
Arzt und Auto
}

Mein Kollege erschien neulich viel zu spät zur Intervision. Völlig untypisch für ihn, zumal er sonst immer zuverlässig und besonders pünktlich ist. Die Erklärung kam, als er erschöpft doch noch erschien: sein Auto hatte ihn im Stich gelassen.

Er ist Psychiater und arbeitet vorwiegend ärztlich-psychotherapeutisch. Voll Enthusiasmus hatte er vor über zehn Jahren seine Praxis eröffnet, ohne altes Geld oder Eigenkapital, jedoch inspiriert von seinen frisch aufblühenden Kindern und den für ihn neuen Herausforderungen als selbständiger Unternehmer und freischaffender Familienernährer.

Die Banken waren grosszügig interessiert an akademisch-medizinischer Kundschaft und stellten ihm werbend Konti zur Verfügung, von denen er sich zur Einrichtung und zum Aufbau der Praxis bedienen könne. In gewohnter Bescheidenheit kaufte er einen Gebrauchtwagen und das für eine Psychiatriepraxis zugegeben einfache Mobiliar. Computer waren damals noch nicht selbstverständlich und daher machte es fast den Anschein von einem gewissen Luxus, als er sich einen PC leistete samt Fax- und Kopiergerät. Insgesamt klassische Praxisstartinvestitionen. In der ländlichen Umgebung hatte er immer grossen Andrang von Patienten und sein Betrieb läuft nach wie vor sehr gut.

Umso härter traf es ihn, dass die Idylle nicht hielt: Die Ehe geriet in eine Krise. In seiner Betroffenheit war auch er verleitet, sich erst noch mehr um häusliche Zufriedenstellung und Präsenz zu bemühen, was zwar den Trennungsprozess hinauszögerte, dafür aber den Praxisumsatz gefährlich drückte: ein Lehrstück für Zerreissproben (in der Industrie spricht man von Materialprüfungen) zwischen beruflicher Verpflichtung und familiärer Zuwendung.

Inzwischen ist er geschieden, und die Kinder stehen erfolgreich am Beginn ihrer Ausbildungen. Er selbst ist immer noch ein engagierter Kollege, der weder resigniert noch stur einzig für seine Praxis lebt. Zum Unterschied von mir hat er gelernt, die buchhalterischen Anforderungen und Normen eines KMU (Klein- und Mittelbetriebs, wie er mir übersetzte) genauestens zu erfüllen, samt doppelter Buchhaltung und den immer wieder neuen Anforderungen an den ständig veralternden Praxiscomputer.

In den heutigen Zeiten müssen wir als Mediziner alle die Gratwanderung zwischen vollem Einsatz für individuellen Standardservice an unseren Patienten und gleichzeitigem Vermeiden von Kostenerhöhung vollziehen. Auch hier jonglierte er mindestens durchschnittlich, obschon er mit seiner primär sozialen und nicht mathematischen Einstellung einigen ökonomischen Druck auszuhalten hatte («Materialprüfungen» kannte er ja bereits). Das ging auch so lange gut, als er jung und dynamisch, einwandfrei gesund und fraglos leistungsfähig war (wie auf der Bankreklame). Im Midlifeabschnitt droht ihn nun aber wieder eine ungeplante und scheinbar banale Schwierigkeit aus der Welt der erfolgreichen KMUs zu kippen: früher als vorgesehen schied eben sein Praxisauto aus dem Verkehr. Dafür liegen keine Reserven bereit.

Als Psychiater und Psychotherapeut kann ich natürlich die Misere meines Freundes gut nachempfinden. Ohne in Saus und Braus zu leben, ist er wieder einmal an die Grenzen der Liquidität gelangt. Und die Bank möchte ihm nach all den Jahren nur noch eigennützig und wesentlich unfreundlicher seinen Kontokorrentkredit gewähren. Also sucht er jetzt jemanden, der ein nicht mehr benötigtes Automobil in seiner Garage stehen hat und es selber alters- oder gesundheitshalber nicht mehr fahren, aber doch nicht unnötig der vorzeitigen Verschrottung zuführen möchte.

Dr. med. Sebastian Pfaundler, Bad Ragaz

PS. Ich jedenfalls melde mein Auto jetzt sofort zum nächsten Service an. 